JOURNAL OF

SYNCHROTRON

RADIATION

ISSN 1600-5775

Received 5 November 2017

Accepted 5 March 2018

Edited by Y. Amemiya, University of Tokyo, Japan

₹ Present address: Medical Physics Department, San Raffaele Scientific Institute, Milan, Italy.

Keywords: breast cancer; radiotherapy; synchrotron radiation; Monte Carlo simulation.

\section{Synchrotron radiation external beam rotational radiotherapy of breast cancer: proof of principle}

\author{
Francesca Di Lillo, ${ }^{a}$ Giovanni Mettivier, ${ }^{a} *$ Roberta Castriconi, ${ }^{a} \ddagger$ Antonio Sarno, ${ }^{a}$ \\ Andrew W. Stevenson, ${ }^{\mathrm{b}, \mathrm{c}}$ Chris J. Hall, ${ }^{\mathrm{b}}$ Daniel Häusermann ${ }^{\mathrm{b}}$ and Paolo Russo ${ }^{\mathrm{a}}$ \\ àDipartimento di Fisica 'Ettore Pancini', Università di Napoli Federico II and INFN Sezione di Napoli, Via Cinthia, \\ Napoli I-80126, Italy, ${ }^{\mathbf{b}}$ Australian Synchrotron, 800 Blackburn Road, Clayton, Victoria 3168, Australia, and ${ }^{\mathbf{c} C S I R O}$ \\ Manufacturing, Private Bag 10, Clayton South, Victoria 3169, Australia. *Correspondence e-mail: mettivier@na.infn.it
}

The principle of rotational summation of the absorbed dose for breast cancer treatment with orthovoltage X-ray beams was proposed by J. Boone in 2012. Here, use of X-ray synchrotron radiation for image guided external beam rotational radiotherapy treatment of breast cancer is proposed. Tumor irradiation occurs with the patient in the prone position hosted on a rotating bed, with her breast hanging from a hole in the bed, which rotates around a vertical axis passing through the tumor site. Horizontal collimation of the X-ray beam provides for whole breast or partial breast irradiation, while vertical translation of the bed and successive rotations allow for irradiation of the full tumor volume, with dose rates which permit also hypofractionated treatments. In this work, which follows a previous preliminary report, results are shown of a full series of measurements on polyethylene and acrylic cylindrical phantoms carried out at the Australian Synchrotron, confirmed by Geant 4 Monte Carlo simulations, intended to demonstrate the proof of principle of the technique. Dose measurements were carried out with calibrated ion chambers, radiochromic films and thermoluminescence dosimeters. The photon energy investigated was $60 \mathrm{keV}$. Image guidance may occur with the transmitted beam for contrast-enhanced breast computed tomography. For a horizontal beam collimation of $1.5 \mathrm{~cm}$ and rotation around the central axis of a $14 \mathrm{~cm}$-diameter polyethylene phantom, a periphery-to-center dose ratio of $14 \%$ was measured. The simulations showed that under the same conditions the dose ratio decreases with increasing photon energy down to $10 \%$ at $175 \mathrm{keV}$. These values are comparable with those achievable with conventional megavoltage radiotherapy of breast cancer with a medical linear accelerator. Dose painting was demonstrated with two off-center 'cancer foci' with $1.3 \mathrm{~Gy}$ and $0.6 \mathrm{~Gy}$ target doses. The use of a radiosensitizing agent for dose enhancement is foreseen.

\section{Introduction}

The purpose of this work, which follows preliminary reports by the team at University and INFN Napoli (De Lucia et al., 2016; Di Lillo et al., 2016a, 2017), was to provide a proof of principle of the technique of external beam rotational radiotherapy with synchrotron radiation $\left(\mathrm{SR}^{3} \mathrm{~T}\right)$ for breast cancer, with the patient in the prone position. Recently, Di Lillo et al. (2017) reported the first analysis of part of an extensive set of data acquired at the Australian Synchrotron in Melbourne, Australia, in a series of experiments in June 2016. Here, in particular, we report on the full set of Monte Carlo (MC) simulations and measurements on phantoms for evaluation of radial dose profiles, 'skin-to-tumor' dose ratio, and nonuniform dose distributions (dose painting applications), as further investigative steps of the $\mathrm{SR}^{3} \mathrm{~T}$ project. A variety of dosimeters were used for in-phantom dose distribution 
assessment: ionization chamber (IC), radiochromic film and thermoluminescence dosimeters (TLDs).

The following sections describe the background, origins and rationale of the $\mathrm{SR}^{3} \mathrm{~T}$ technique.

\subsection{Background}

Radiotherapy treatment is considered to be the standard of care for breast cancer in women, for whom it represents the most common malignancy (Torre et al., 2015). The commonly used technique for breast cancer is megavoltage photon 3Dconformal radiotherapy (3D-CRT), using a medical linear accelerator: the therapy ( 45 to 50 Gy in 25 fractions of 1.8 to 2 Gy followed by a boost of 10 to $16 \mathrm{~Gy}$ ) is normally delivered in 6 to 6.5 weeks with two opposed tangential fields to encompass the breast with the patient in supine position, often including part of the underlying chest wall and the lower axilla. X-ray irradiation at $6 \mathrm{MV}$ energy produces a so-called buildup effect whereby the dose to the surface tissue layers several millimeters below the skin is reduced to a fraction of the maximum dose at depth in the tissue, so avoiding skin tissue complications after therapy.

With improved survival after breast radiotherapy, more patients will be at risk of long-term radiation-induced toxicity, thus making it even more important to reduce the dose to all organs at risk. Indeed, when irradiating the breast or the chest wall, it is unavoidable that close organs like the lung and the heart receive a small fraction (e.g. several percent) of the total dose to the clinical target. Critical issues are the associated increased rate of major coronary events, heart disease and lung disease. After breast cancer radiotherapy, the average dose for whole lung may be $5.7 \mathrm{~Gy}$ and $4.4 \mathrm{~Gy}$ for whole heart (Taylor et al., 2017). Coronary and heart disease after radiotherapy is especially of concern in patients treated for leftsided breast cancer. As the rate of the ischemic heart disease or lung disease is proportional to the mean dose to the organ, the goal is to reduce the dose to the heart and lung as much as possible. Positioning the patient prone on the linac couch may reduce the dose to the chest and the heart, the pendant breast being in this way kept away from the chest wall.

Intensity modulated radiotherapy (IMRT) with photons has been investigated in order to improve the dose homogeneity and to reduce the doses to surrounding organs at risk. Photon planning using 3D-techniques, deep inspiration breath-hold and prone position are implemented to minimize cardiopulmonary dose for patients. However, the radiation dose to the heart remains relatively high, even with the use of advanced photon-based techniques (Mast et al., 2014). Due to its ballistic physical characteristics (high dose localization and high cell-killing effect at the Bragg peak of the energy deposition curve), proton beam therapy (PBT) may eventually enable a further decrease of the dose to the heart. A proton beam is characterized by a dose distribution that is finite and adjustable in depth depending on the beam energy. For this reason, $\mathrm{PBT}$ is increasingly being investigated to treat breast cancer showing a considerable reduction of the mean heart dose with respect to 3D-CRT and IMRT (MacDonald, 2016).
To address the question of whether proton therapy can meaningfully decrease radiation-induced cardiovascular toxicities in breast cancer, in early 2016 the Patient-Centered Outcomes Research Institute-funded Radiotherapy Comparative Effectiveness (RadComp) was started: a pragmatic randomized trial of proton therapy versus photon therapy for patients with nonmetastatic breast cancer (Verma et al., 2016).

For early-stage breast cancer, partial breast irradiation (PBI) is being investigated as a means to limit the dose delivery to the tissue volume surrounding the tumor bed with respect to irradiating the whole breast. PBI is performed with a hypofractionated, high-dose delivery, with respect to fractionated dose delivery to the whole breast with low-doses per fraction. Stereotactic body radiation therapy (SBRT), where large radiation doses are delivered in a few fractions, is also under investigation for breast cancer, in particular using the Cyberknife frameless radiosurgery system at $6 \mathrm{MV}$. A cobalt60 unit for stereotactic partial breast radiotherapy has been recently introduced (FDA, 2017), where the patient's prone position and the use of a vacuum-assisted cup to immobilize the breast may help reducing radiation-induced side effects.

\subsection{Kilovoltage external beam radiotherapy with an orthovoltage $\mathrm{X}$-ray tube}

In 2012, in a pioneering paper (Prionas et al., 2012), J. Boone and his team at University of California Davis set forth the idea of performing kilovoltage external-beam radiotherapy $(\mathrm{kV}$-EBRT) for breast cancer. The dedicated setup employs an orthovoltage X-ray tube operating at $320 \mathrm{kVp}(\mathrm{kVp}=$ peak kilovoltage) and rotating in full circles around the breast, rather than a megavoltage $\mathrm{X}$-ray beam from a medical linac irradiating the breast with two tangential beams, as in conventional breast radiotherapy with photons.

In the case of left-sided breast cancer, with the patient in the prone position and hence with the breast laying away from the chest wall, $\mathrm{kV}$-EBRT might be investigated also with the purpose of exploring ways for reducing the dose to organs at risk.

Kilovoltage radiotherapy for treating non-superficial lesions is only a recent field of investigation. Indeed, the technology for MV X-ray beam delivery of radiation dose to deep-seated tumors is widespread and well advanced. On the other hand, the technology of low-energy X-rays $(<300 \mathrm{keV})$ produced by X-ray tubes, for conformal dose delivery to tumors deep into the body, is just under development by few groups. These studies follow the technology advancements and positive clinical outcome of (modulated) arc therapy techniques in MV radiotherapy occurring in the last ten years, whose clinical use for breast cancer is still limited (Cozzi et al., 2017). In particular, further clinical evidence is necessary to demonstrate the dosimetric benefits of volumetric modulated arc therapy of breast cancer as regards the fractionation scheme, the prone positioning, the irradiation trajectory (Cozzi et al., 2017). 
A recent MC study (Bazalova-Carter et al., 2017; Breitkreutz et al., 2017) explored the use of kilovoltage X-ray arc therapy at $200 \mathrm{kV}$ for conformal radiotherapy, with limited arc or full circle rotation of the source, showing the feasibility of the technique for lesions up to $4 \mathrm{~cm}$ in diameter to depths of $8-10 \mathrm{~cm}$ in head- or body-sized phantoms. This technique, however, is intended for a large $(e . g .30 \mathrm{~cm} \times 30 \mathrm{~cm})$ beam produced by a $200 \mathrm{kV}$ kilovoltage arc therapy X-ray source consisting of a large tungsten anode, a type of source still to be produced.

The kV-EBRT technique (Prionas et al., 2012) could be implemented on the rotating gantry of a cone-beam computed tomography $(\mathrm{CT})$ scanner dedicated to the breast [see, for example, the recent review by Sarno et al. (2015)], for example, as realized by the Davis group and operating typically at $80 \mathrm{kVp}$, thus providing precise three-dimensional localization of the tumor mass in the breast volume. The advantages of the $\mathrm{kV}$-EBRT technique with respect to conventional radiotherapy were described as a simplified and more economical setup (which also does not require a bunker as for a linac), the potential for reduced patient repositioning error and improved treatment accuracy in fractionated radiotherapy, the use of a prone rather than a supine patient position.

The well known effect of absorbed dose buildup of 6$10 \mathrm{MV}$ photon beams, which assures dose sparing to the surface tissues in conventional radiotherapy, with a maximum dose at $15 \mathrm{~mm}$ below the zero-depth layer in tissue at $6 \mathrm{MV}$, is much reduced for the $320 \mathrm{kVp}$ X-ray beam adopted in Boone's setup. Indeed, for orthovoltage X-ray beams, the nominal depth of maximum dose beneath the patient's surface is essentially zero (Podgorsak, 2005). On the other hand, for $6 \mathrm{MV}$ X-ray photons the dose buildup effect produces a surface dose as low as $15 \%$ of the maximum depth dose (Podgorsak, 2005). Despite the practical unavailability of the buildup region for kilovoltage photons, the principle of rotational summation leads to higher dose at the tumor site, where the axis of rotation of the gantry is positioned, by suitably collimating the beam, so that substantial skin tissue sparing could be obtained. On the other hand, there are some limits of $\mathrm{kV}$-EBRT related to the relatively low dose rate available at practical distances $(50-100 \mathrm{~cm})$ from the collimator units of an orthovoltage tube (of the order of 0.1-1 Gy $\mathrm{min}^{-1}$ ) and to the thick lead collimator for a $320 \mathrm{kVp}$ beam, which introduces penumbra and scatter. The technique of $\mathrm{kV}$-EBRT with orthovoltage X-ray tubes has been validated experimentally at $120 \mathrm{kVp}$ by the Davis group (Prionas et al., 2012) and at $300 \mathrm{kVp}$ by the Naples group (De Lucia et al., 2016).

Although the principle of $\mathrm{kV}$-EBRT is conceptually simple and cost-effective, the available studies are limited to MC investigations and laboratory validations, and a clinically compatible setup for patient irradiation on a dedicated breast CT platform is not available yet. Key technological issues are the realization of dynamically adjustable beam collimators, $\mathrm{X}$-ray tube selection and positioning for irradiation of lesions close to the chest wall, and suitable rotating/translating couches.

\subsection{Radiotherapy with synchrotron radiation}

At the state of the art, synchrotron stereotactic radiotherapy (SSRT) (Renier et al., 2008; Bräuer-Krisch et al., 2015), minibeam radiation therapy (MBRT) (Deman et al., 2012) and microbeam radiation therapy (MRT) (Crosbie et al., 2013; Bravin et al., 2015; Grotzer et al., 2015; Poole et al., 2017) are the radiotherapy techniques under investigation at several synchrotron radiation (SR) facilities. These include the Australian Synchrotron (AS) in Melbourne, Australia, and the European Synchrotron Radiation Facility (ESRF) in Grenoble, France, having active programs for developing clinical applications in radiotherapy.

The main interest of synchrotron radiation therapy (SRT) studies to date is the brain tissue. However, a feasibility study of the use of the monochromatic MRT (m-MRT) technique at 60 and $100 \mathrm{keV}$ for breast cancer treatment was recently reported in the literature (Wysokinski et al., 2016). This study, carried out at the Canadian Light Source (CLS) in Saskatoon, Canada, showed that the m-MRT for the treatment of the cancerous tissue slowed down the tumor growth implanted in mice after irradiation compared with the untreated controls and that the irradiation induced cancer cell apoptosis by triggering a stress response of the cells.

The availability of world-class facilities and the associated expertise of the investigators enable studies that have the potential to substantially improve SRT for cancer and advance our understanding of fundamental tumor biology. The combination of SRT with drugs and/or other substances that enhance radiation effects, at optimized and tunable X-ray energies, as well as its combination with gene-mediated therapies must be tested in order to establish whether these lead to considerable improvements compared with conventional radiotherapy alone.

\subsection{External beam rotational radiotherapy with $S R$ for breast cancer}

Recently, the Naples group envisaged the possibility of the new technique of external beam rotational radiotherapy of breast cancer using SR (De Lucia et al., 2016; Di Lillo et al., 2016a, 2017). In SR ${ }^{3} T$, the patient is lying prone on a rotating/ translating bed, with her breast hanging from a hole in the bed, while the SR beam (suitably collimated in the horizontal and vertical directions) irradiates the organ, incident from a plurality of azimuthal angles (Fig. 1). A full rotation of the bed around a vertical axis centered at the tumor site permits the highest dose to be delivered at the isocenter and a reduced dose at the entrance and exit surfaces. A combination of successive horizontal and vertical translations of a robotized bed, interleaved by $360^{\circ}$ rotations, permits a full irradiation of the tumor volume. Whole breast irradiations can be performed by opening the horizontal collimators to several centimeters and realizing a series of turns with the axis of rotation shifted laterally, so producing an almost flat dose profile covering the size of the breast diameter.

Our in-house-developed $\mathrm{MC}$ code for $\mathrm{SR}^{3} \mathrm{~T}$ [described in this work and by Di Lillo et al. (2017)] showed that a skin 


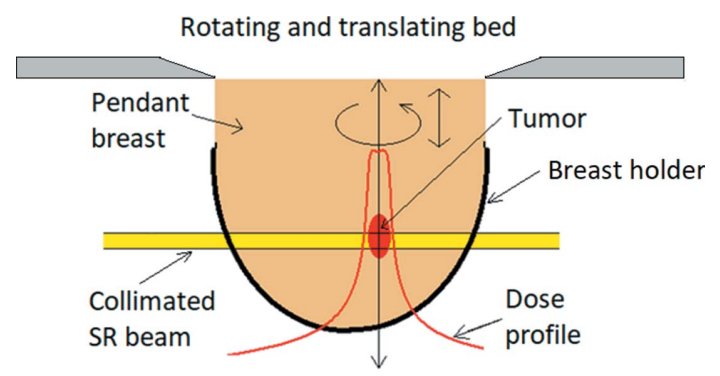

Figure 1

Sketch illustrating the $\mathrm{SR}^{3} \mathrm{~T}$ technique. The patient is prone on a bed with her breast hanging from a hole. The bed is rotating $360^{\circ}$ around a vertical axis centered at the tumor site, while the collimated kilovoltage X-ray beam from an SR source irradiates the tumor. The bed is then translated vertically and horizontally and additional rotational scans are performed, in order to conform the treatment to the tumor volume. Rotational summation assures a tumor-to-skin dose ratio similar to conventional megavoltage radiotherapy. A dose enhancement at the tumor site might be obtained by using a radiosensitizer (e.g. gold nanoparticles).

sparing effect can be produced in such a radiotherapy technique with photon energies down to $60 \mathrm{keV}$. Since dose rates largely exceeding $10 \mathrm{~Gy} \mathrm{~min}^{-1}$ (considered adequate for breast radiotherapy) can be obtained at SR sources, irradiation of the tumor (partial irradiation) or of the whole organ (total irradiation) of the female breast for total doses of the order of tens of grays can be performed in one or a few fractions. The beam energy will be selected in the range 60$200 \mathrm{keV}$, after extensive MC simulations. Rotational summation at the tumor where the axis of rotation is positioned will produce dose summation up to the prescribed level in the target tumor tissue, while the dose to the skin would be limited to levels of the order of one-tenth of the tumor dose. At the same time, low dose levels may be delivered to healthy internal organs and tissues including lung, heart and carotid arteries, due to the limited photon scatter dose toward the chest wall. This is estimated to be less than $5 \%$ of the tumor dose by Prionas et al. (2012) for a $320 \mathrm{kVp}$ beam. The threedimensional localization of the tumor before as well as during the RT procedure with SR will be performed via parallel-beam CT imaging of the transmitted X-ray beam with the patient in the prone position (Lindfors et al., 2008; O'Connell et al., 2010; Russo et al., 2010; Mettivier \& Russo, 2011; Mettivier et al., 2011; Sarno et al., 2015) on the robotized support. This will implement SR breast CT (Longo et al., 2016; Mettivier et al., 2016; Sarno et al., 2016a) for the needs of image-guided radiotherapy of breast cancer. Using kilovoltage beams, on the other hand, will permit the use of an iodinated contrast medium (commonly adopted clinically for contrast-enhanced digital mammography) or other radiosensitizers (e.g. gold nanoparticles; Lin et al., 2015) for realizing contrast-enhanced breast CT as well as dose-enhanced radiotherapy. This technique exploits the increase of the absorbed energy in the tumor where the iodinated agent accumulates. The increased photoelectric absorption at energies in the 60-200 keV range, with respect to $\mathrm{kV}$-EBRT at $320 \mathrm{kVp}$ or conventional radiotherapy with $6 \mathrm{MV}$ beams, is expected to provide a higher dose enhancement effect.

\section{Materials and methods}

\subsection{Experimental assessment}

We conducted the experiment for the feasibility study of $\mathrm{SR}^{3} \mathrm{~T}$ at the Imaging and Medical Beamline (IMBL) of the AS in Clayton (Melbourne), Australia (http:// www.synchrotron.org.au/aussyncbeamlines/imaging-medical). AS is a third-generation synchrotron facility with a circumference of $216 \mathrm{~m}$ operating in 'top-up' mode at a nominal electron energy of $3 \mathrm{GeV}$ and a ring current of $200 \mathrm{~mA}$. The r.m.s. electron beam size in the straight sections is $320 \mu \mathrm{m}$ horizontally and $16 \mu \mathrm{m}$ vertically, corresponding to a Gaussian FWHM of $754 \mu \mathrm{m}$ and $38 \mu \mathrm{m}$, respectively (Stevenson et al., 2010, 2017). IMBL produces a fan beam of polychromatic radiation from a superconducting multi-pole wiggler (SCMPW) with 60 pole pairs $(52 \mathrm{~mm}$ period) and a peak magnetic field of 4.0 T (Pelliccia et al., 2016; Stevenson et al., 2017). A double-crystal Laue monochromator (DCLM), with the center at a distance of $16.2 \mathrm{~m}$ from the source, is used to select a single energy up to $120 \mathrm{keV}$ (Stevenson et al., 2012). IMBL has three optical hutches (1A, 2A and $3 \mathrm{~A})$ and three experimental hutches $(1 \mathrm{~B}, 2 \mathrm{~B}$ and $3 \mathrm{~B})$. In each hutch, it is possible to work with monochromatic or pink photon beam.

The measurements were performed in the hutch 3B, situated in a dedicated satellite building providing a source-tosample distance of approximately $140 \mathrm{~m}$. In this hutch the SR beam, which can reach a size of $500 \mathrm{~mm} \times 40 \mathrm{~mm}(\mathrm{H} \times \mathrm{V})$, has a divergence of $3.8 \mathrm{mrad}$ in the horizontal direction and $0.3 \mathrm{mrad}$ in the vertical direction. We carried out this study at a single photon energy of $60 \mathrm{keV}$, selected as the maximum energy achievable in the hutch $3 \mathrm{~B}$ to obtain a large $\mathrm{X}$-ray beam with an intensity variation of less than $8 \%$ along a $160 \mathrm{~mm}$ length in the horizontal direction. The magnetic field of SCMPW was $3.0 \mathrm{~T}$.

\subsection{Dosimeters calibration and beam uniformity assessment}

As dosimeters for air kerma measurements performed freein-air or in a phantom, we used a $100 \mathrm{~mm}$-long pencil IC (model 20X6-3CT, Radcal Corp., Monrovia, CA, USA, read out by a Radcal 2026C dosimeter), radiochromic films (GafChromic ${ }^{\circledR}$ EBT3 and XR-QA2, Ashland, KY, USA) and TLD-100 LiF thermoluminescence dosimeters.

The response of the pencil IC in the monoenergetic laminar beam at $60 \mathrm{keV}$ was calibrated in terms of air kerma in air with reference to a free-in-air parallel-plate IC (model ADC IC105) employed at the IMBL beamline (Crosbie et al., 2013; Stevenson et al., 2017). For calibration, the pencil chamber was scanned vertically through the SR beam at a constant speed (Prezado et al., 2011) and temperature and pressure correction was applied. The parallel chamber was operated at $2 \mathrm{kV}$ without windows. In order to obtain a different air kerma rate, layers of copper with different thicknesses attenuated the intensity of photon beam. We estimated that the contribution of harmonics $(180 \mathrm{keV})$ for SCMPW $(3.0 \mathrm{~T})$ was of the order of $1 \%$.

The response of the EBT3 (lot \#12011401) radiochromic films was calibrated in terms of air kerma evaluated in a 

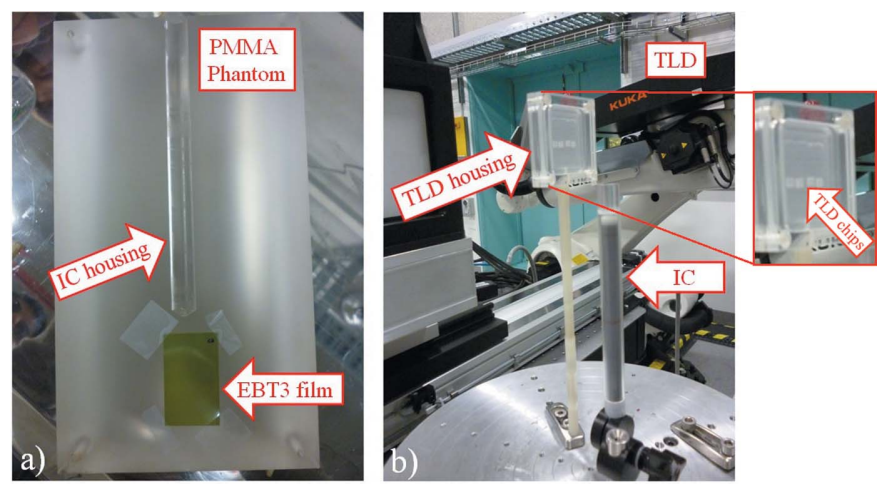

Figure 2

(a) Photograph showing the midplane face of the two-halves cylindrical PMMA phantom containing a cavity for the IC, and with the EBT3 film inserted in place. (b) Setup for TLD irradiation: four TLD chips inserted in the PMMA housing and $100 \mathrm{~mm}$ pencil IC placed along the vertical direction. The inset shows the four chips in place in the housing.

polymethylmethacrylate (PMMA) phantom in the range 0.052 Gy, while the response of the XR-QA2 films (lot \#10261501) was calibrated in terms of air kerma free-in-air in the range $0.005-0.200 \mathrm{~Gy}$. We used the pencil IC to measure the air kerma. A PMMA cylindrical phantom $(14 \mathrm{~cm}$ diameter, $15 \mathrm{~cm}$ height), composed of two semi-cylinders to permit the film placement and with a cylindrical hole (13 $\mathrm{mm}$ diameter) along the cylinder axis, was employed for the EBT3 calibration. During irradiation, the pencil chamber was allocated in the hollow cavity along the cylinder axis, while the film piece was at the midplane of the cylinder, at $1 \mathrm{~cm}$ from the IC to avoid scatter radiation contamination (Fig. 2a). The dosimeters placed in the PMMA phantom (for EBT3 calibration) or freein-air (for XR-QA2 calibration) were translated along the beam height direction at a constant speed because of the laminar beam configuration (Prezado et al., 2011; Brown et al., 2012). The scanning method mimics a uniform irradiation of dosimeters. Different exposures were obtained by setting different scan speeds. Films were digitized in RGB scanning mode (72 dpi, 48-bit, TIFF image) with an Epson Perfection V750 Pro flat-bed scanner and their response in the red channel was evaluated. Following Devic et al. (2016) and Di Lillo et al. (2016b), the calibration curve was calculated for EBT3 and XR-QA2 films, respectively.

The irradiation protocol for TLD100s calibration was similar to that used for the radiochromic films. The TLD chips placed in a PMMA housing (Fig. 2b) (1 mm PMMA upstream and $1 \mathrm{~mm}$ downstream) were exposed to the SR beam adopting the scanning method (Emiro et al., 2015). The dosimeter response was calibrated in terms of air kerma free-in-air measured with the pencil chamber in the range $0.5-1.7 \mathrm{~Gy}$. For each calibration point, the average response of four different TLDs was evaluated.
A preliminary assessment of the beam uniformity was also performed by imaging the SR beam with a radiochromic film, Gafchromic $^{\circledR}$ XR-QA2. Following Di Lillo et al. (2015), the film response was converted to photon fluence per unit air kerma $\left(\mathrm{mm}^{-2} \mathrm{mGy}^{-1}\right)$ providing the two-dimensional distribution of the beam intensity in a plane transverse to the beam axis.

\subsection{Radial dose profile measurements}

The radial dose distribution was evaluated in a polyethylene (PE) cylindrical phantom (14 cm diameter, $15 \mathrm{~cm}$ height) with the $100 \mathrm{~mm}$ pencil IC. The phantom had cylindrical holes (diameter of $1.3 \mathrm{~cm}$ ) placed at different distances from the cylinder axis (Figs. $3 a$ and $3 b$ ) to insert the IC. Air kerma was measured by irradiating the IC at each position during a complete rotation of the phantom. The PE cylinder was placed on an assembly of PMMA slabs $(20 \mathrm{~cm} \times 20 \mathrm{~cm} \times 18 \mathrm{~cm})$ mimicking the chest wall (Fig. $3 c$ ). The axis of rotation coincided with the cylinder axis. The radial dose distribution was evaluated with an SR beam collimated to $0.8 \mathrm{~cm}$ vertically and to $1.5,4,7$ or $15 \mathrm{~cm}$ horizontally.

\subsection{Assessment of the periphery-to-center dose ratio}

We estimated the periphery-to-center dose ratio in a $14 \mathrm{~cm}$ diameter PMMA cylindrical phantom by measuring the air kerma at the phantom axis (center) and at $4 \mathrm{~cm}$ from the axis (periphery) during a complete rotation of the phantom. Air kerma measurements were made with both EBT3 radiochromic film and TLD-100. The cross section of the SR beam was $1.5 \mathrm{~cm}(\mathrm{H}) \times 1.6 \mathrm{~cm}(\mathrm{~V})$.

A cylinder with two $13 \mathrm{~mm}$-diameter cavities was used for measurements with TLD-100. Ten TLD chips filling the cavity were irradiated at each position and the average of their response was used to calculate the dose ratio.

Radiochromic films were placed at the midplane of the same phantom used for the calibration. The dose ratio was calculated by estimating the mean pixel value, calibrated in terms of air kerma, in two regions of interest (ROIs) (center and periphery) of area $3 \mathrm{~mm} \times 3 \mathrm{~mm}$.
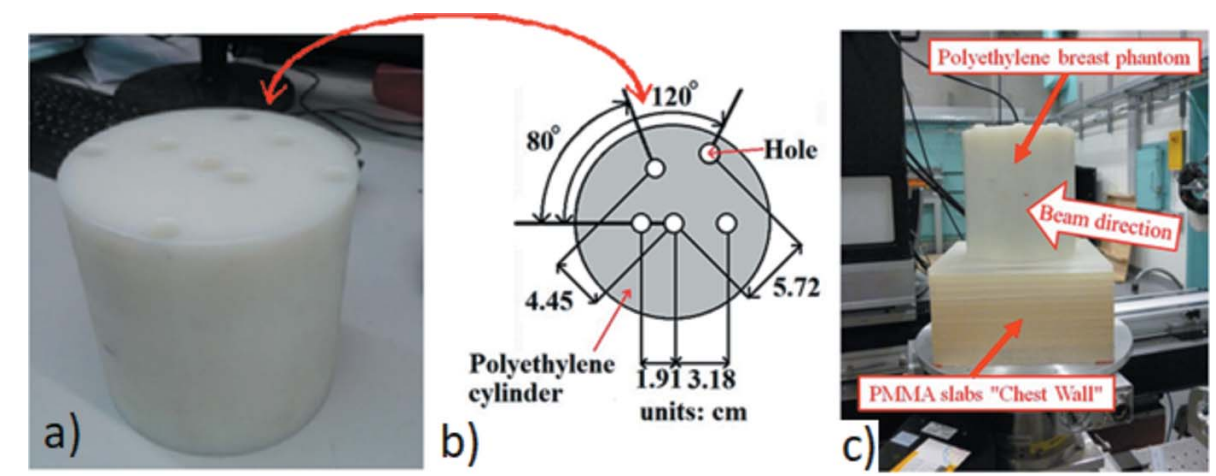

Figure 3

(a) Photograph and (b) scheme of the polyethylene cylindrical phantom with five cylindrical holes at various radial distances for hosting a $100 \mathrm{~mm}$-long ion chamber. (c) Experimental setup for radial dose profile measurement. In this image the IC is positioned in the central hole of the phantom. 


\subsection{Dose painting application}

The feasibility of dose painting applications was studied by performing measurements with radiochromic film. Pieces of EBT3 radiochromic film of size $10 \mathrm{~cm} \times 5 \mathrm{~cm}(\mathrm{H} \times \mathrm{V})$ were positioned at the midplane of a $14 \mathrm{~cm}$-diameter PMMA cylinder phantom placed at the isocenter. A collimated SR beam of size $15 \mathrm{~mm} \times 16 \mathrm{~mm}(\mathrm{H} \times \mathrm{V})$ irradiated the phantom at an air kerma rate free-in-air of $2.22 \mathrm{~Gy} \mathrm{~min}^{-1}$. Non-uniform dose distributions, i.e. two off-center foci and a line distribution, were obtained by multiple rotations of the phantom and shifting the axis of rotation laterally. In particular, the two offcenter foci were irradiated by shifting the axis of rotation by $4.9 \mathrm{~cm}$. The rotation speed was set at $10^{\circ} \mathrm{s}^{-1}$ for the first turn (for delivering $0.6 \mathrm{~Gy}$ to the first focus) and at $5^{\circ} \mathrm{s}^{-1}$ for the second turn (with 1.2 Gy delivered to the second focus). The line dose distribution was obtained by rotating the phantom three times at a speed of $10^{\circ} \mathrm{s}^{-1}$ and shifting laterally the center of rotation by $0.75 \mathrm{~cm}$ at each rotation.

\subsection{MC simulations}

The experimental results were compared with those obtained via MC simulations. We reproduced the experimental irradiation geometries with an MC code developed inhouse, based on the Geant4 toolkit (version 10.00). The MC code, originally developed for breast dosimetry in mammography (Sarno et al., 2016b,c, 2017a) and digital breast tomosynthesis (Sarno et al., 2017b), was implemented for breast radiotherapy application with an SR beam. A preliminary validation of this new version of the MC code is reported by Di Lillo et al. (2017). We simulated a rectangular X-ray source that generated the monoenergetic and laminar beam. The sizes of the X-ray source were varied according to the simulated case. The rotational irradiation was reproduced by rotating the source along a $360^{\circ}$ circle, in steps of $1^{\circ}$, around a PE (density $=0.9325 \mathrm{~g} \mathrm{~cm}^{-3}$ ) or a PMMA (density = $\left.1.19 \mathrm{~g} \mathrm{~cm}^{-3}\right)$ cylindrical phantom $(14 \mathrm{~cm}$ diameter, $15 \mathrm{~cm}$ height). The scored energy included the energy deposited in the material by both primary and secondary photons (scattered photons, fluorescent and bremsstrahlung photons) during the interaction with material and by the electrons produced at the photon hit (multiple scatter included). In addition, the event position was scored. A three-dimensional dose map of $1 \mathrm{~mm} \times 1 \mathrm{~mm} \times 1 \mathrm{~mm}$ voxels was obtained associating the deposited energy by any interaction with the event position.

The results from MC simulations for a beam of size $150 \mathrm{~mm}$ $\times 1 \mathrm{~mm}(\mathrm{H} \times \mathrm{V})$ were compared with those obtained with a simple model at 60, 80, 100, 120 and $180 \mathrm{keV}$. This 'simple model' calculates the dose deposited purely on the basis of an attenuated primary X-ray beam and the relevant geometrical conditions which prevail. That is, the X-ray beam incident on the dosimeter is assumed to be the primary beam after allowance for attenuation on the basis of photoelectric, coherent (Rayleigh) and incoherent (Compton) cross sections, e.g. no allowance is made for any scatter contributions which reach the dosimeter. The input parameters are: the width and any lateral offset of the X-ray beam; incident dose rate (which could be essentially air kerma or absorbed dose rate for water, and so on) and the associated mass energy-absorption coefficient; mass attenuation coefficient and density for the phantom material; diameter of the (cylindrical) phantom; mass energy-absorption coefficient and density for the material corresponding to the dosimeter; diameter of the (cylindrical) dosimeter and its (radial) distance from the center of the phantom; number of full rotations of the phantom and the associated angular speed. If the number of full rotations is set to zero, the static dose rate is calculated, provided that the angular position of the dosimeter is also specified. The derived formula involves a double integral with respect to lateral position across the dosimeter and time, which cannot be solved analytically and so is evaluated numerically. The values of the attenuation and absorption coefficients are derived by logarithmic interpolation of values from the NIST database (Hubbell \& Seltzer, 1995).

\section{Results}

\subsection{Dosimeter calibration}

Figs. 4 and 5 show the calibration curves of the dosimeter's response at $60 \mathrm{keV}$. A linear fit function was employed for the calibration of the pencil chamber response in terms of air kerma rate and for the calibration of the TLD's response in air kerma free-in-air. The film response evaluated in terms of net optical density (net OD) for EBT3 and net reflectance change (net $\Delta R$ ) for XR-QA2 was correlated by means of a power function with the air kerma measured in the PMMA phantom and air kerma free-in-air, respectively.

\subsection{SR beam uniformity}

Fig. 6 shows the image of the SR beam acquired with radiochromic film XR-QA2 (Fig. 6a) and the corresponding horizontal line profile (Fig. $6 b$ ). The SR beam size was $15 \mathrm{~cm}$ $\times 1.6 \mathrm{~cm}(\mathrm{H} \times \mathrm{V})$. For comparison, the calculated 'Roll-off' curve at $60 \mathrm{keV}$ is shown together with the measured profile. The maximum variation of the beam flux along the horizontal direction was about $6 \%$.

\subsection{Radial dose profile assessment with the IC}

The radial dose profile in the PE phantom was evaluated by measuring the air kerma with the $100 \mathrm{~mm}$ pencil chamber for beam widths from 1.5 to $15 \mathrm{~cm}$. The experimental setup reported in $\$ 2.3$ was reproduced with the MC code. In order to take into account the length of the pencil chamber, the dose integrated along $100 \mathrm{~mm}$ in the direction of the cylinder axis was evaluated for the simulated data. Fig. 7 shows a comparison between the dose profiles measured in the phantom (symbol) and profiles evaluated via MC simulation (line) at beam widths of 1.5, 4, 7 and $15 \mathrm{~cm}$ and a fixed beam height of $8 \mathrm{~mm}$. The agreement in terms of mean percentage difference is within $5 \%$. The data show that the shape of the radial dose profile depends on the SR beam width. In particular, for a $15 \mathrm{~cm}$ beam width the dose distribution is flat. When the beam 

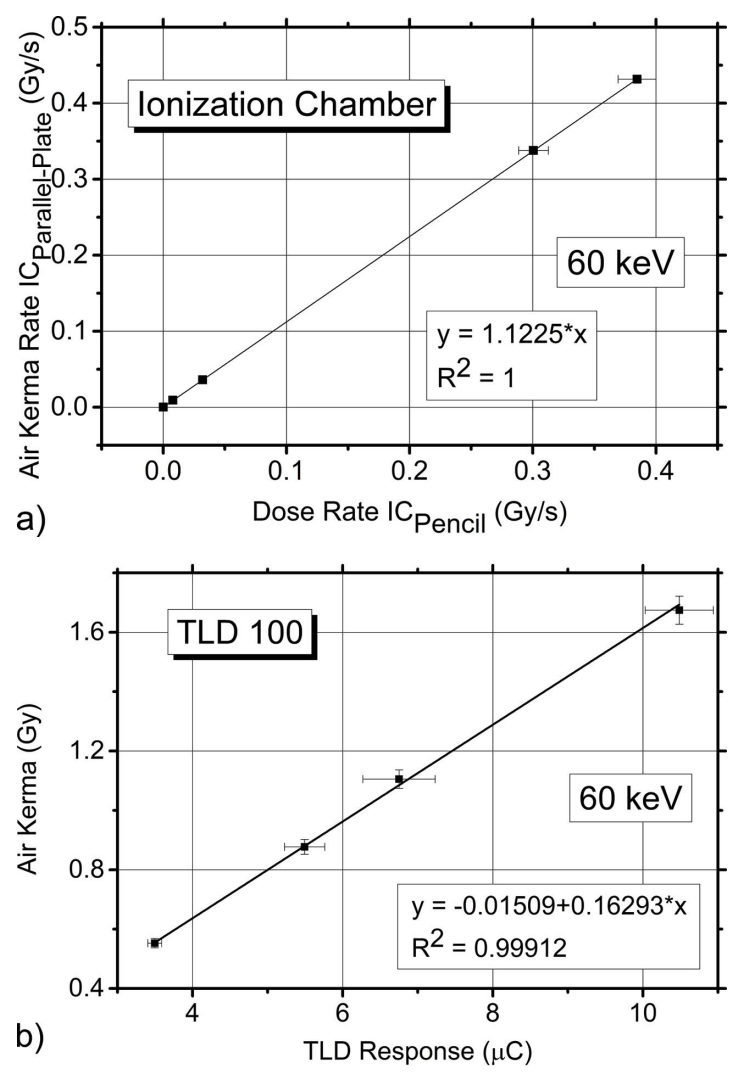

Figure 4

Calibration curve for the response of $(a)$ the pencil IC and (b) TLD dosimeters at $60 \mathrm{keV}$. The continuous line is a linear fit to the data points.

width is progressively narrowed, the radial profile becomes peaked at the center of rotation. A periphery-to-center dose ratio of $103 \%, 48 \%, 33 \%$ and $17 \%$ is estimated via $\mathrm{MC}$ simulation for beam widths of $15,7,4$ and $1.5 \mathrm{~cm}$, respectively.

The dose distributions shown in Fig. 7 include two components: one due to the energy released in the volume directly irradiated with the $8 \mathrm{~mm}$-height beam and a second component due to the energy deposited by the scatter radiation in the phantom volume not directly exposed to the beam. It is of interest to evaluate the extent of the two separate terms in the total dose. Hence, we carried out MC simulations in which we derived the radial profile of the dose distribution in the $14 \mathrm{~cm}$ diameter PE phantom due to each separate contribution. Fig. 8 shows the radial dose distribution obtained by scoring the energy only in the irradiated volume of the phantom (corresponding to ROI 1 in Fig. 9), or in the zone outside the irradiated volume (reached by scattered photons) (corresponding to ROI $2+$ ROI 3 in Fig. 9), or in the whole volume (corresponding to ROI $1+$ ROI 2 + ROI 3 in Fig. 9), for a collimated beam width of $1.5 \mathrm{~cm}$ (Fig. $8 a$ ) or for a wide beam of $15 \mathrm{~cm}$ width (Fig. 8b). The dose was expressed in percent of the central dose.

For the collimated beam (Fig. $8 a$ ), the dose profile in the irradiated volume is peaked at the center of rotation showing a dose distribution different from that evaluated in the whole volume. The dose profile in the un-irradiated volume has a triangular shape with a maximum value at the rotation center.
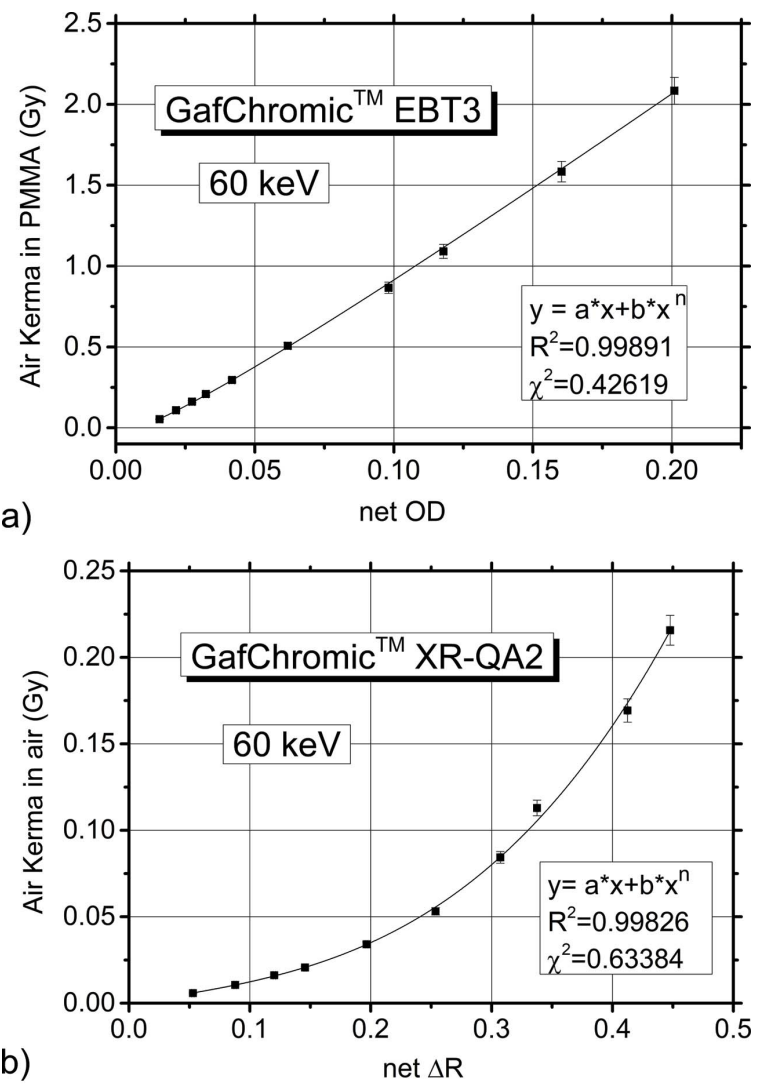

Figure 5

Dose-response curves evaluated for (a) EBT3 and (b) XR-QA2 radiochromic films at $60 \mathrm{keV}$. The power function indicated on the graphs was employed to fit the experimental data.
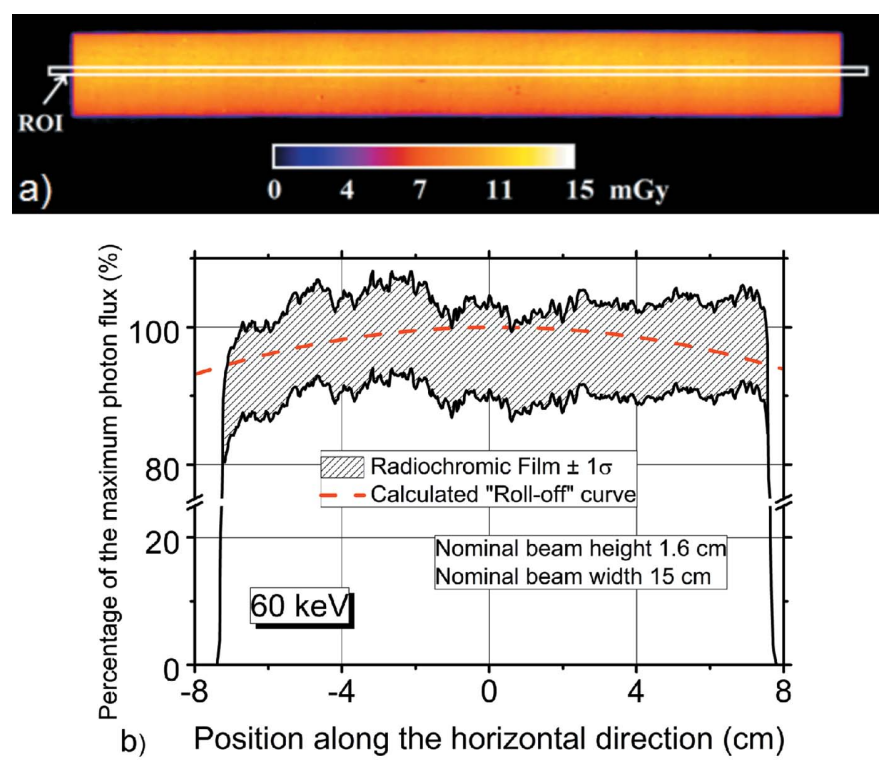

Figure 6

(a) Dose map of the SR beam at $60 \mathrm{keV}$ acquired with XR-QA2 radiochromic film. The film response was calibrated in terms of air kerma in air. (b) Horizontal average profile in the ROI shown in (a) at $60 \mathrm{keV}$ evaluated in the radiochromic film dose map. The maximum horizontal variation is about $6 \%$. 


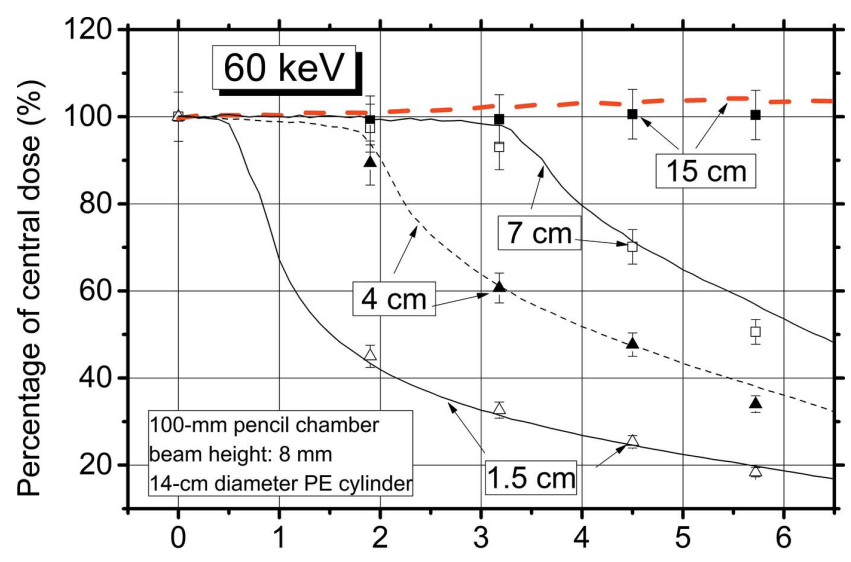

Distance from the axis of the cylindrical phantom $(\mathrm{cm})$

Figure 7

Comparison between the measured (symbols) and simulated (lines) relative air kerma in a $14 \mathrm{~cm}$-diameter PE cylindrical phantom for beam width of $1.5,4,7$ and $15 \mathrm{~cm}$. For the MC simulation, the dose distribution was evaluated by integrating the dose along $100 \mathrm{~mm}$ in the direction of the cylinder axis.

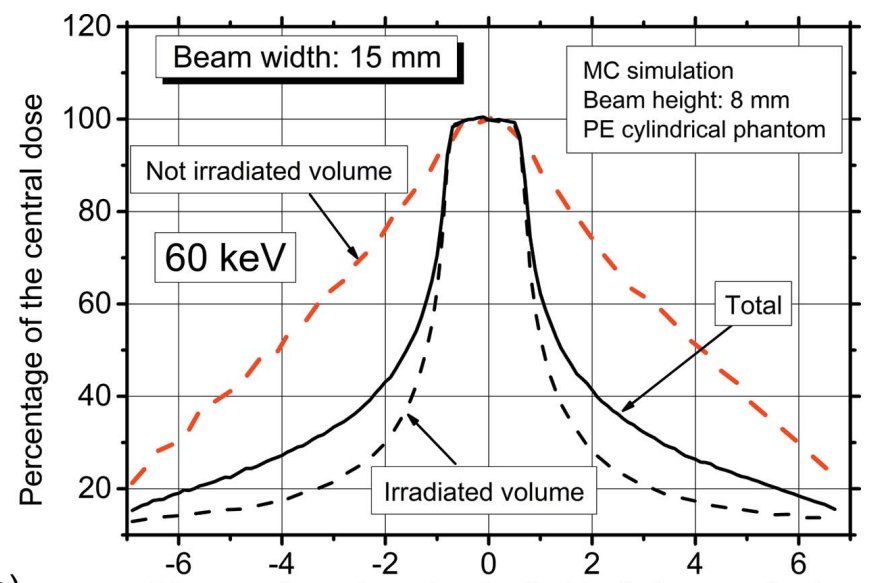

a)

Distance from the axis of cylindrical phantom $(\mathrm{cm})$

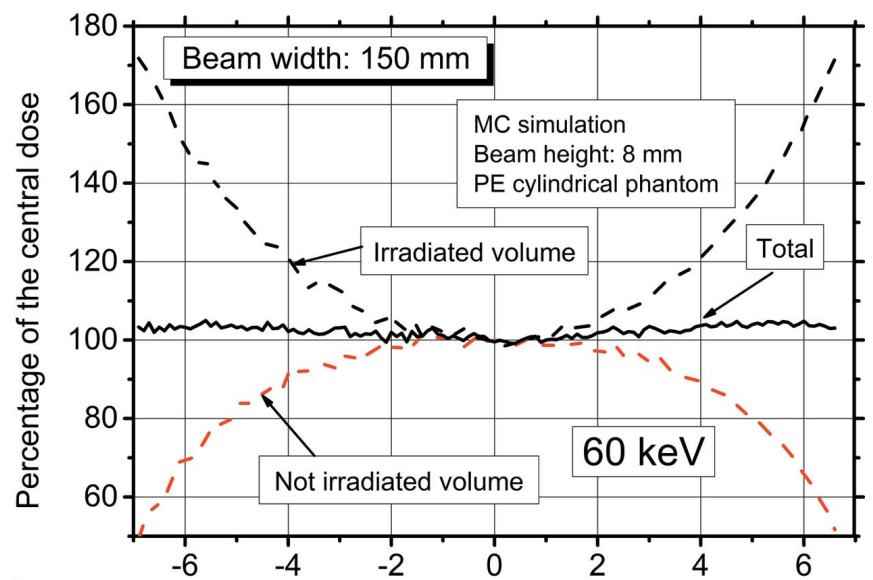

b) Distance from the axis of the cylindrical phantom $(\mathrm{cm})$

Figure 8

Dose profile along a diameter, obtained via $\mathrm{MC}$ simulation in a $14 \mathrm{~cm}-$ diameter PE cylindrical phantom for a beam width of $(a) 1.5 \mathrm{~cm}$ and (b) $15 \mathrm{~cm}$. For each beam collimation, the dose profile was evaluated in the irradiated volume, in the not irradiated volume and in the whole volume used for the measurements.

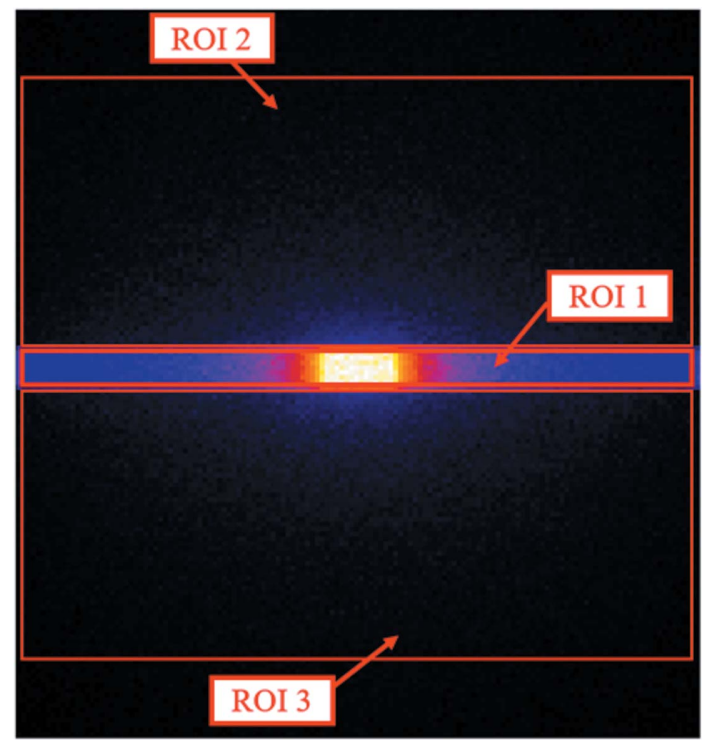

Figure 9

Two-dimensional map of the energy released in the $14 \mathrm{~cm}$ PE cylindrical phantom. The map was obtained via $\mathrm{MC}$ simulation with a beam collimation of $1.5 \mathrm{~cm} \times 0.8 \mathrm{~cm}(\mathrm{H} \times \mathrm{V})$. We show the ROIs corresponding to the volume where the dose distributions reported in Fig. 8 were estimated.

The irradiation with the wide beam (Fig. $8 b$ ) produces a cupped dose distribution in the irradiated volume because of the exponential attenuation of the X-ray beam in the material, which provides a greater dose deposit at the periphery. A domed profile characterizes the dose distribution in the unirradiated volume and the combined effect of these two contributions results in a flat dose distribution in the total volume.

We report the radial distributions of the dose deposited in the irradiated volume for different beam widths (from 1.5 to $15 \mathrm{~cm}$ ) at $60 \mathrm{keV}$ (Fig. 10). A periphery-to-center dose ratio of $167 \%, 59 \%, 34 \%$ and $14 \%$ is estimated for beam width collimations of $15,7,4$ and $1.5 \mathrm{~cm}$.

\subsection{Skin sparing effect}

The MC code was employed to study the skin sparing effect obtainable with the $\mathrm{SR}^{3} \mathrm{~T}$ technique at energies in the range 60-175 keV. In particular, the center-to-periphery dose ratio in a $\mathrm{PE}$ phantom (14 cm diameter and $15 \mathrm{~cm}$ height) was estimated at $60,80,100,120$ and $175 \mathrm{keV}$. The beam size was set at $1.5 \mathrm{~cm} \times 0.6 \mathrm{~cm}(\mathrm{H} \times \mathrm{V})$. Fig. 11 shows the center-toperiphery dose ratio as a function of photon energy. We observed an exponential increment of the dose ratio by increasing the photon energy, from 7 at $60 \mathrm{keV}$ to 10 at $175 \mathrm{keV}$.

\subsection{Comparison of MC simulations with the simple method}

A comparison between the dose distribution obtained with MC code (which takes into account primary and scatter fields) and the simple method (only primary) is shown in Fig. 12. The 


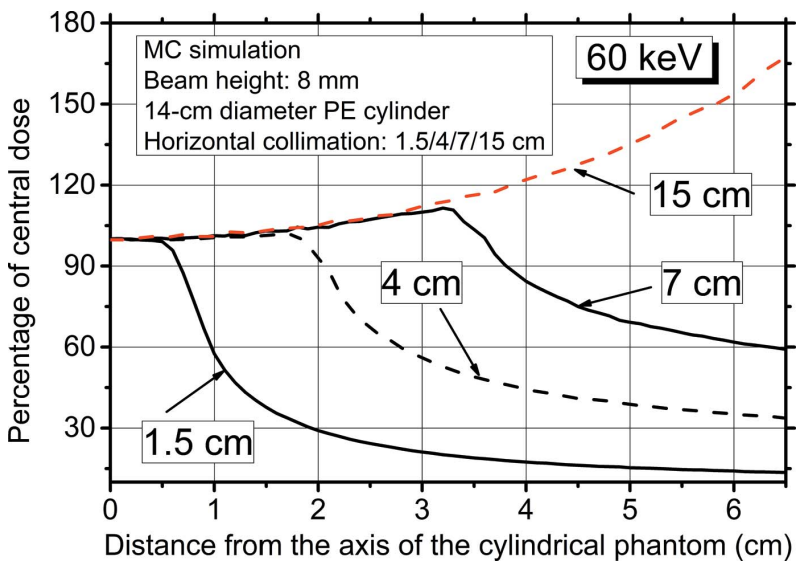

Figure 10

Dose profile obtained via MC simulation in a $14 \mathrm{~cm}$-diameter PE cylindrical phantom, for various widths of the horizontal collimation (from $1.5 \mathrm{~cm}$ to $15 \mathrm{~cm}$ ). The dose profile was evaluated in the irradiated phantom slice.

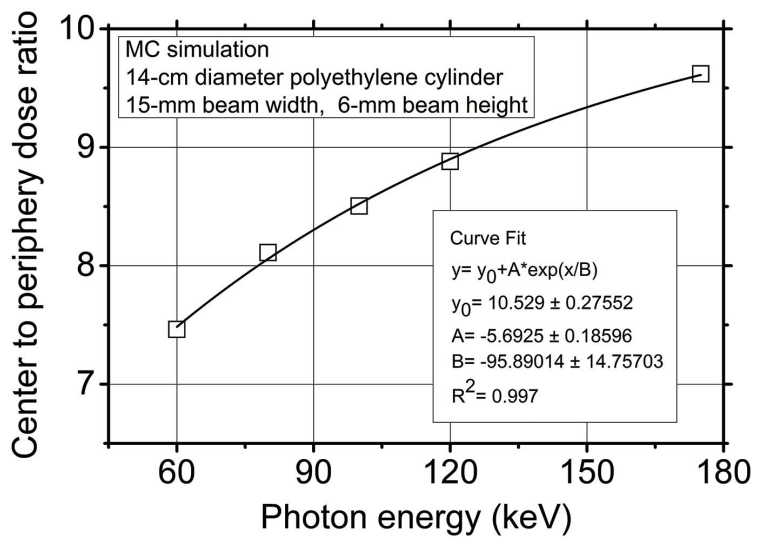

Figure 11

Center-to-periphery dose ratio as a function of photon energy, estimated via $\mathrm{MC}$ simulation in a $14 \mathrm{~cm}$ PE cylindrical phantom. The beam is collimated to $15 \mathrm{~mm} \times 6 \mathrm{~mm}$, and the photon energy is $60,80,100,120$ and $175 \mathrm{keV}$. The continuous line is an exponential fit to the data points.

beam energy was tuned from 60 to $180 \mathrm{keV}$. For the data from the $\mathrm{MC}$ simulation, we scored the energy deposited in the volume of the phantom directly irradiated by the X-ray beam. The simple model produces dose distributions similar to those from MC simulations. A maximum deviation of about $1.5 \%$ was calculated. We point out that in Fig. 12 the $y$-axis is a ratio, so that only relative variations between the different models are shown.

\subsection{Radial dose profile assessment with TLD and radiochromic film}

Table 1 reports the periphery-to-center dose ratio at $4 \mathrm{~cm}$ from the cylinder axis measured with EBT3 radiochromic film and with TLDs. A dose ratio of $24 \% \pm 3 \%$ was estimated with TLD chips, in agreement with simulations results within the experimental error. However, a dose ratio of $47 \% \pm 3 \%$ was evaluated with radiochromic film. We investigated this discrepancy and attributed it, at least in part, to the process
Table 1

Periphery-to-center dose ratio simulated with MC code and measured with TLD-100 and EBT radiochromic film for a SR beam collimated at $1.5 \mathrm{~cm}$ along the horizontal direction at $60 \mathrm{keV}$.

\begin{tabular}{ll}
\hline & $\begin{array}{l}\text { Periphery-to-center } \\
\text { dose ratio (\%) }\end{array}$ \\
\hline EBT3 radiochromic film & $47 \pm 3$ \\
TLD-100 & $24 \pm 3$ \\
MC simulation & $21.2 \pm 0.3$ \\
\hline
\end{tabular}

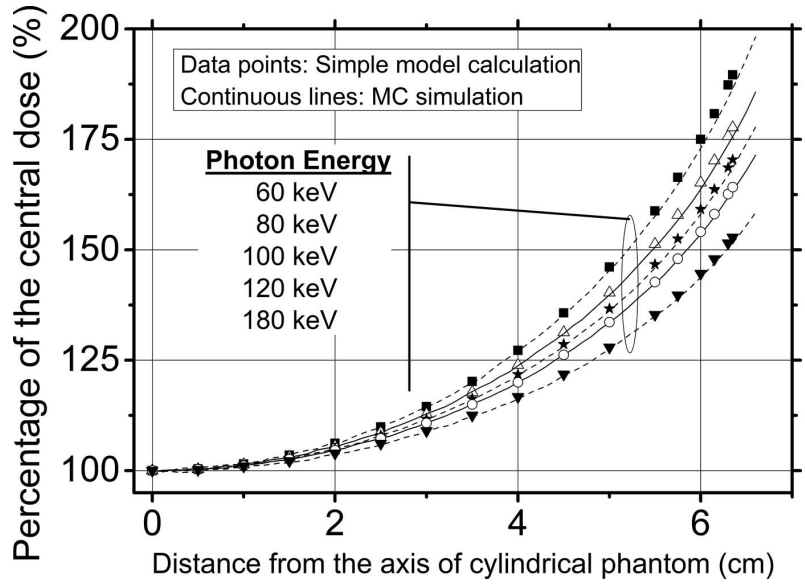

Figure 12

Dose profiles along a diameter of the PE cylindrical phantom, obtained via scatter-free simple model calculation (data points) or via $\mathrm{MC}$ simulations (lines), for a monoenergetic beam at $60 \mathrm{keV}, 80 \mathrm{keV}, 100 \mathrm{keV}$, $120 \mathrm{keV}$ or $180 \mathrm{keV}$.

of film scanning. The films were scanned with the scanner EPSON V750 PRO which has been in use in our laboratory for many years. We noticed the onset of an artefact with excess pixel values on one side of the scanning field, which may produce alteration of the line profile measured with the film dosimeters.

\subsection{Dose painting}

Figs. 13 and 14 show the non-uniform dose distributions (two off-center foci and line dose distribution) evaluated with measurements (Fig. 13) and with MC simulations (Fig. 14), respectively. The surface plots show the map of the percentage of the maximal dose. The irradiation of two off-center foci, obtained with two successive rotations by shifting the center of rotation, resulted in two target doses of about $1.2 \mathrm{~Gy}$ and $0.6 \mathrm{~Gy}$, respectively, with the low-dose focus near the center of rotation. Both the experimental and the $\mathrm{MC}$ data show a flat distribution of the dose inside the low-dose focus. On the other hand, the dose increases by increasing the distance from the phantom center inside the other focus, as resulting from the X-ray attenuation in the phantom (Fig. 15a). The overlapping of the two distributions produces a dose deposition of about $30 \%$ of the maximal dose at the edge nearest the highdose focus. At the opposite edge, measurements and MC simulation provide two different values of dose: $10 \%$ and $20 \%$ of the maximal dose, respectively. 

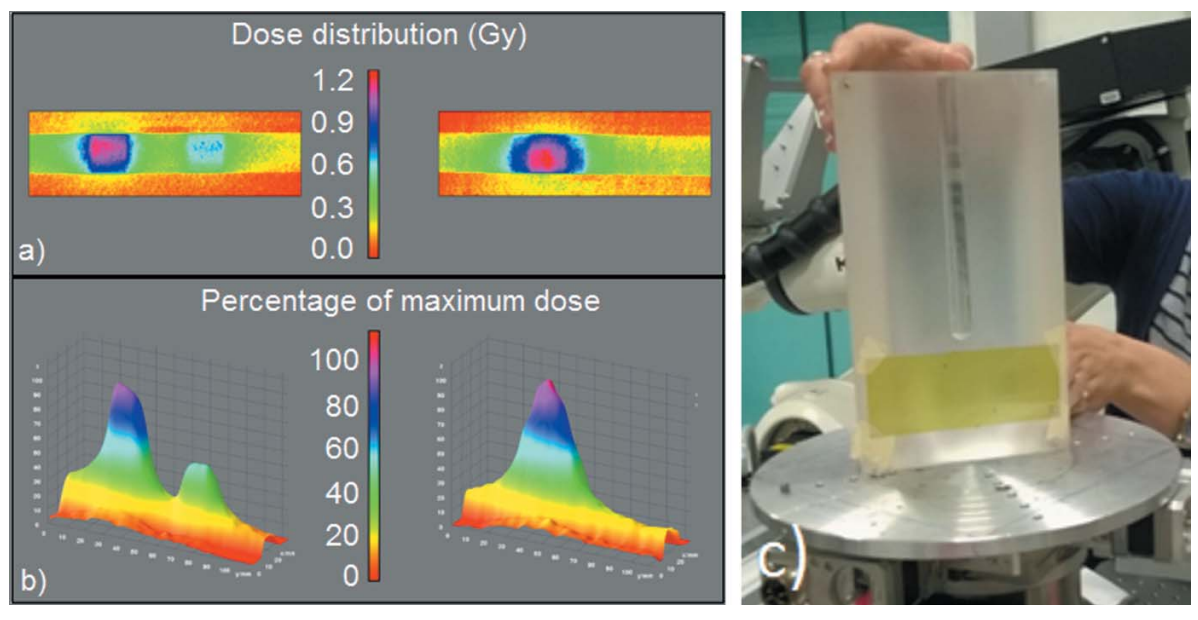

Figure 13

(a) Two-dimensional dose distributions evaluated with an EBT3 radiochromic film in a $14 \mathrm{~cm}$ diameter PMMA phantom and $(b)$ corresponding surface plots of percentage of maximum dose: two off-center foci (left side) and line distribution (right side). The intended dose distribution was obtained with multiple rotations. (c) For ease of visualization of the sample geometry, photograph taken during the placement of one-half of the cylindrical phantom on the rotation stage, where the piece of EBT3 film is visible at the mid-plane in the PMMA phantom. Above the film the longitudinal half cavity realized for hosting the ion chamber is visible, along the vertical axis of the cylindrical phantom.

For line dose distribution, measurements indicate a dose deposition of $22 \%$ of the maximal dose at $3 \mathrm{~cm}$ from the peak of the dose distribution, while a dose value of $28 \%$ is calculated at the same distance with data from the MC simulation (Fig. 15b).

We point out that, although the dose profiles obtained with the two different data sets (measurements and MC simula-

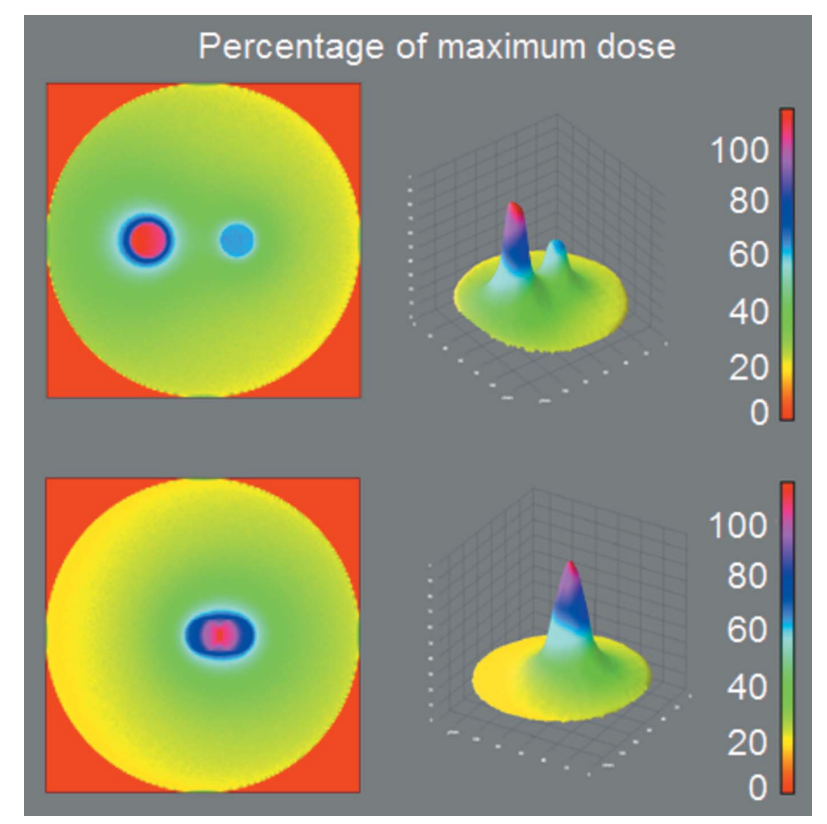

Figure 14

Irradiation of two off-center foci and line dose distributions in a $14 \mathrm{~cm}$ diameter PMMA phantom obtained via MC simulations and line dose profiles evaluated in terms of percentage of maximum dose.

Figure 15 tions) demonstrated similar shapes, the percentage of the maximal dose is systematically overestimated by the simulated data with respect to the experimental data (Fig. 15). Also in this case, the disagreement could be due to the radiochromic film response.

\section{Discussion}

Prionas et al. (2012) proposed to use a collimated X-ray beam rotating around the breast in order to irradiate a focal tumor target with $\mathrm{X}$-rays from an orthovoltage X-ray tube. Via MC simulations, they showed that a $178 \mathrm{keV}$ photon beam produces the same depthdose characteristics in a $14 \mathrm{~cm} \mathrm{PE}$ cylinder phantom as obtained with a $320 \mathrm{kVp}$ spectrum. In this work, following the preliminary investigation reported by Di Lillo et al. (2017), with measurements performed at AS and MC simulations, we have provided a
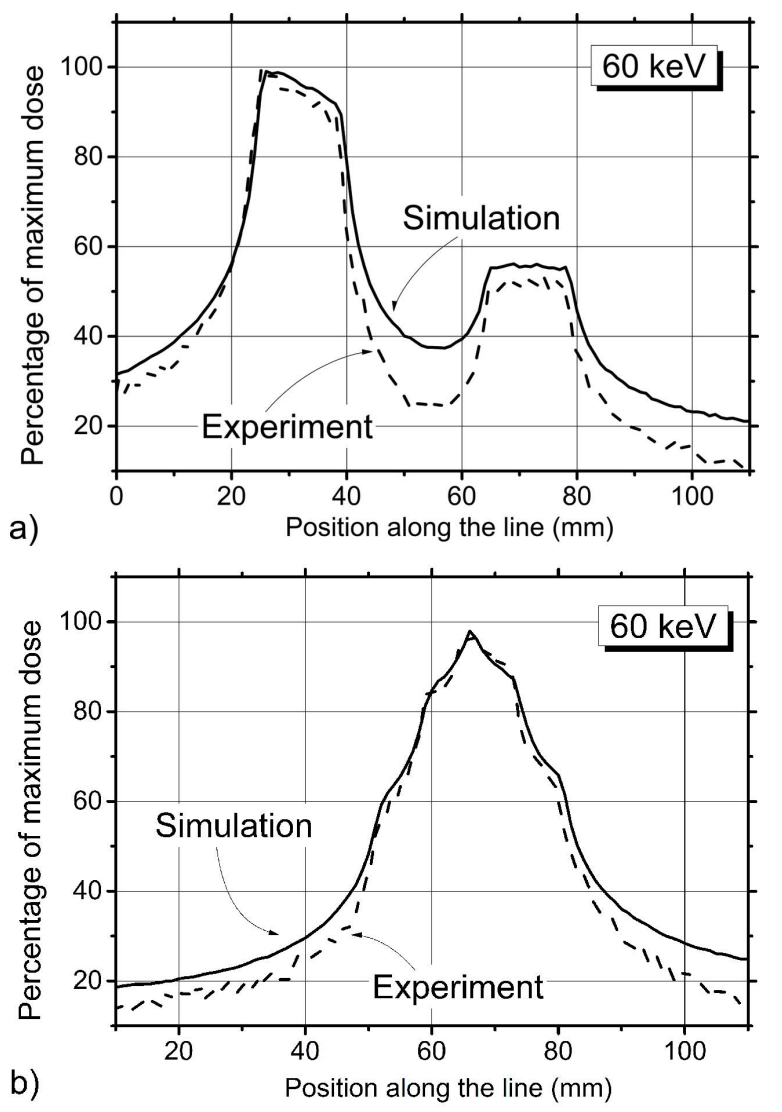

Line dose profiles of the percentage of maximum dose for $60 \mathrm{keV}$ irradiation for $(a)$ two off-center foci and $(b)$ a linear distribution. Dashed line: experimental; continuous line: simulated data. 
proof-of-principle showing the feasibility of the external beam radiotherapy for breast cancer treatment with SR at low energy $(60 \mathrm{keV})$. We showed that the shape of the dose distribution in a PE cylinder phantom depends on the SR beam collimation. The beam collimation in the direction perpendicular to the phantom axis produces a dose distribution peaked in the center of rotation. In particular, we showed that it is possible to obtain a focus of dose in the center of rotation with a 'tumor-to-skin' dose ratio of about $14 \%$ of the target dose (evaluated at $60 \mathrm{keV}$, for a beam collimation of $1.5 \mathrm{~cm}$ and the center of rotation corresponding to the tumor position, on the cylinder axis). This value is comparable with that $(10 \%)$ obtained via $\mathrm{MC}$ simulation at $175 \mathrm{keV}$, under the same irradiation conditions. This energy value produces the same depth-dose distribution produced by an orthovoltage $\mathrm{X}$-ray tube operating at $300 \mathrm{kVp}$. For comparison, Prionas et al. (2012) reported dose to the skin of $<7 \%$ of the maximal dose for a $320 \mathrm{kVp} \mathrm{X}$-ray beam collimated at $1 \mathrm{~cm}$.

We showed that the dose distributions from MC simulations are comparable with the ones obtained for a simple model with a wide beam. A similar result was also obtained by Boone et al. (2009), for collimated beams ( $1 \mathrm{~cm}$ and $2 \mathrm{~cm}$ width). It is of interest to point out that the discrepancy between the two distributions increases by increasing the beam height because of the greater contribution of the scatter radiation.

The evaluation of the periphery-to-center dose ratio with TLDs and EBT3 radiochromic films showed a discrepancy of about $20 \%$. The value estimated with TLD at $4 \mathrm{~cm}$ from the cylinder axis was comparable with that obtained via MC simulation.

We demonstrated the dose painting feasibility with both MC simulations and measurements with radiochromic film dosimeters, at a synchrotron radiation beamline. Non-uniform dose distributions can be obtained by using multiple rotations, by shifting the center of rotation and changing the rotation speed. In particular, we reproduced a double off-center foci and a line dose distribution. The comparison between simulated data and experimental data acquired with radiochromic films showed a systematic overestimation of simulated data with respect to the measurements. This result is in the opposite direction with respect to that obtained for the evaluation of the periphery-to-center dose ratio, where the simulation provides an underestimate of this value with respect to that provided by radiochromic films. This disagreement indicates the need to improve the study of EBT3 response to X-ray beam at low energy for the irradiation condition used for $\mathrm{SR}^{3} \mathrm{~T}$, also using the new led-lamp scanner EPSON V850 PRO recently available in our laboratory. Although we showed the proof of principle of dose painting, the constraints stressed by Prionas et al. (2012), i.e. beam collimation accuracy, lesion proximity to the skin surface and chest wall, and total treatment time, are still present.

The results obtained at $60 \mathrm{keV}$ provide encouragement to examine the use of low-energy photon beams with a doseenhancement agent. The availability of a monoenergetic X-ray beam with high dose rate combined with the use of possible dose-enhancement agents (e.g. iodinated solution or gold nanoparticles) would permit the breast irradiation to be optimized by selecting the best SR beam energy. In particular, the use of low photon energy (e.g. $200 \mathrm{keV}$ or lower) could increase the photoelectric absorption and hence increase the dose deposit in the region of accumulation of the radiosensitizer.

We note that the radiotherapy technique presented in this work can be implemented also using a polyenergetic SR beam. Indeed, white and pink SR beams are available at the IMBL of AS, at the ID17 beamline of ESRF and the BMIT beamline of CLS: these beams can provide kilovoltage photons with a higher total photon flux than that of the monoenergetic beam, well suited for the application of the proposed technique. This would require calibration of the dosimeters (radiochromic films and ICs) for the specific polyenergetic beam adopted for the treatment.

In addition, image guided $\mathrm{SR}^{3} \mathrm{~T}$ (with or without contrast enhancement) can be performed (in propagation-based phasecontrast imaging, for example with a fine-pitch photoncounting pixel detector; Longo et al., 2016) permitting realtime CT imaging with additional imaging-related glandular doses of the order of a few mGy (Mettivier et al., 2016). Potential applications of this technique include limited fractions rotational radiotherapy, radiotherapy boost and small lesion irradiation. A specific application could be 'synchrotron radiation radiotherapy surgery' for treatment of small size breast lesions.

As reported by Di Lillo et al. (2017), the dose rates available at the synchrotron facility are comparable with, or higher than, the dose rates used in conventional radiotherapy with a MV $\mathrm{X}$-ray beam of $5 \mathrm{~Gy} \mathrm{~min}^{-1}$. It is important to take into account that the SR beam is fixed in space and therefore the bed hosting the patient needs to translate and rotate (in multiple turns where necessary) in order to irradiate the breast lesion. This movement could produce the irradiation of healthy portions of the organ. A breast holder similar to that proposed for breast CT (Sarno et al., 2015) or a thermoplastic mask such as is used for brain radiotherapy could be employed to reduce this effect. The total treatment times for a future $\mathrm{SR}^{3} \mathrm{~T}$ session could be of order $10 \mathrm{~min}$, taking into account a reference rotation time of $1 \mathrm{~min}$, the necessity of multiple rotations/ translations, and the high dose rate available at the facilities.

\section{Conclusions}

On the basis of MC simulations and experiments at $60 \mathrm{keV}$ irradiating breast phantoms at the Australian Synchrotron, we have presented a proof of concept of the $\mathrm{SR}^{3} \mathrm{~T}$ technique for breast cancer treatment with kilovoltage photons from a collimated synchrotron radiation source. The principle of rotational summation of the absorbed dose at the site of the target volume was verified, showing the possibility of surface dose reduction up to $14 \%$ at $60 \mathrm{keV}$. The tunability of the radiation source will be exploited in future experiments for investigating the dependence of the tumor-to-skin dose ratio on the photon energy. Additional studies will be performed also to investigate the use of a dose enhancement agent. 


\section{References}

Bazalova-Carter, M., Weil, M. D., Breitkreutz, D. Y., Wilfley, B. P. \& Graves, E. E. (2017). Med. Phys. 44, 597-607.

Boone, J. M., Prionas, N., Burkett, G. W., Packard, N. J. \& Yang, K. (2009). Med. Phys. 36, 2747.

Bräuer-Krisch, E., Adam, J. F., Alagoz, E., Bartzsch, S., Crosbie, J., DeWagter, C., Dipuglia, A., Donzelli, M., Doran, S., Fournier, P., Kalef-Ezra, J., Kock, A., Lerch, M., McErlean, C., Oelfke, U., Olko, P., Petasecca, M., Povoli, M., Rosenfeld, A., Siegbahn, E. A., Sporea, D. \& Stugu, B. (2015). Phys. Med. 31, 568-583.

Bravin, A., Olko, P., Schültke, E. \& Wilkens, J. J. (2015). Phys. Med. 31, 561-563.

Breitkreutz, D. Y., Weil, M. D., Zavgorodni, S. \& Bazalova-Carter, M. (2017). Med. Phys. 44, 6548-6559.

Brown, T. A. D., Hogstrom, K. R., Alvarez, D., Matthews, K. L., Ham, K. \& Dugas, J. P. (2012). Med. Phys. 39, 7412-7417.

Cozzi, L., Lohr, F., Fogliata, A., Franceschini, D., De Rose, F., Filippi, A. R., Guidi, G., Vanoni, V. \& Scorsetti, M. (2017). Radiat. Oncol. 12, 200.

Crosbie, J. F., Rogers, P. A. W., Stevenson, A. W., Hall, C. J., Lye, J. E., Nordström, T., Midgley, S. M. \& Lewis, R. A. (2013). Med. Phys. 40, 062103.

De Lucia, P. A., Mettivier, G., Di Lillo, F., Sarno, A. \& Russo, P. (2016). Phys. Med. Suppl. 32, 19.

Deman, P., Vautrin, M., Edouard, M., Stupar, V., Bobyk, L., Farion, R., Elleaume, H., Rémy, C., Brabier, E. L., Estève, F. \& Adam, J. F. (2012). Int. J. Radiat. Oncol. Biol. Phys. 82, 693-700.

Devic, S., Tomic, N. \& Lewis, D. (2016). Phys. Med. 32, 541-556.

Di Lillo, F., Dreossi, D., Emiro, F., Fedon, C., Longo, R., Mettivier, G., Rigon, L., Russo, P. \& Tromba, G. (2015). J. Instrum. 10, C05002.

Di Lillo, F., Mettivier, G., Sarno, A., Castriconi, R. \& Russo, P. (2017). Phys. Med. 41, 20-25.

Di Lillo, F., Mettivier, G., Sarno, A. \& Russo, P. (2016a). Phys. Med. 32(Suppl. 3), 253-254.

Di Lillo, F., Mettivier, G., Sarno, A., Tromba, G., Tomic, N., Devic, S. \& Russo, P. (2016b). Med. Phys. 43, 583-588.

Emiro, F., Di Lillo, F., Mettivier, G., Fedon, C., Longo, R., Tromba, G. \& Russo, P. (2015). Rad. Prot. Dosim. 168, 40-45.

FDA (2017). FDA clears stereotactic radiotherapy system for use in treating breast cancer, https://www.fda.gov/NewsEvents/Newsroom/ PressAnnouncements/ucm590313.htm (accessed 4 February 2018).

Grotzer, M. A., Schültke, E., Bräuer-Krisch, E. \& Laissue, J. A. (2015). Phys. Med. 31, 564-567.

Hubbell, J. H. \& Seltzer, S. M. (1995). Tables of X-ray Mass Attenuation Coefficients and Mass Energy-Absorption Coefficients from $1 \mathrm{keV}$ to $20 \mathrm{MeV}$ for Elements $Z=1$ to 92 and 48 Additional Substances of Dosimetric Interest. NIST Technical Report 5632. NIST, USA.

Lin, Y., Paganetti, H., McMahon, S. J. \& Schuemann, J. (2015). Med. Phys. 42, 5890-5902.

Lindfors, K. K., Boone, J. M., Nelson, T. R., Yang, K., Kwan, A. L. C. \& Miller, D. F. (2008). Radiology, 246, 725-733.

Longo, R., Arfelli, F., Bellazzini, R., Bottigli, U., Brez, A., Brun, F., Brunetti, A., Delogu, P., Di Lillo, F., Dreossi, D., Fanti, V., Fedon, C., Golosio, B., Lanconelli, N., Mettivier, G., Minuti, M., Oliva, P., Pinchera, M., Rigon, L., Russo, P., Sarno, A., Spandre, G., Tromba, G. \& Zanconati, F. (2016). Phys. Med. Biol. 61, 1634-1649.

MacDonald, S. M. (2016). Int. J. Radiat. Oncol. Biol. Phys. 95, 46-48.

Mast, M. E., Vredeveld, E. J., Credoe, H. M., van Egmond, J., Heijenbrok, M. W., Hug, E. B., Kalk, P., van Kempen-Harteveld, L. M. L., Korevaar, E. W., van der Laan, H. P., Langendijk, J. A., Rozema, H. J. A., Petoukhova, A. L., Schippers, J. M., Struikmans, H. \& Maduro, J. H. (2014). Breast Cancer Res. Treat. 148, 33-39.
Mettivier, G., Fedon, C., Di Lillo, F., Longo, R., Sarno, A., Tromba, G. \& Russo, P. (2016). Phys. Med. Biol. 61, 569-587.

Mettivier, G. \& Russo, P. (2011). IEEE Trans. Nucl. Sci. 58, 703-713.

Mettivier, G., Russo, P., Cesarelli, M., Ospizio, R., Passeggio, G., Roscilli, L., Pontoriere, G. \& Rocco, R. (2011). Nucl. Instrum. Methods. A, 629, 350-356.

O'Connell, A., Conover, D. L., Zhang, Y., Seifert, P., Logan-Young, W., Lin, C. F. L., Sahler, L. \& Ning, R. (2010). Am. J. Roentgenol. 195, 496-509.

Pelliccia, D., Poole, C. M., Livingstone, J., Stevenson, A. W., Smyth, L. M. L., Rogers, P. A. W., Haüsermann, D. \& Crosbie, J. C. (2016). J. Synchrotron Rad. 23, 566-573.

Podgorsak, E. B. (2005). Radiation Oncology Physics: A Handbook for Teachers and Students. Vienna: International Atomic Energy Agency.

Poole, C. M., Day, L. R. J., Rogers, P. A. W. \& Crosbie, J. C. (2017). Biomed. Phys. Eng. Expr. 3, 025001.

Prezado, Y., Vautrin, M., Martínez-Rovira, I., Bravin, A., Estève, F., Elleaume, H., Berkvens, P. \& Adam, J. F. (2011). Med. Phys. 38, 1709-1717.

Prionas, N. D., McKenney, S. E., Stern, R. L. \& Boone, J. M. (2012). Int. J. Rad. Oncol. Biol. Phys. 84, 533-539.

Renier, M., Brochard, Th., Nemoz, C., Requardt, H., Bräuer, E., Estève, F., Balosso, J., Suortti, P., Baruchel, J., Elleaume, H., Berruyer, G., Berkvens, P. \& Bravin, A. (2008). Eur. J. Radiol. 68, S147-S150.

Russo, P., Mettivier, G., Lauria, A. \& Montesi, M. C. (2010). IEEE Trans. Nucl. Sci. 57, 160-172.

Sarno, A., Dance, D. R., van Engen, R. E., Young, K. C., Russo, P., Di Lillo, F., Mettivier, G., Bliznakova, K., Fei, B. \& Sechopoulos, I. (2017a). Med. Phys. 44, 3848-3860.

Sarno, A., Masi, M., Antonelli, N., Di Lillo, F., Mettivier, G., Castriconi, R. \& Russo, P. (2017b). IEEE Trans. Radiat. Plasma Med. Sci. 1, 322-328.

Sarno, A., Mettivier, G., Di Lillo, F. \& Russo, P. (2016b). Lect. Notes Comput. Sci. 9699, 190-196.

Sarno, A., Mettivier, G., Di Lillo, F. \& Russo, P. (2016c). Phys. Med. Biol. 62, 306-325.

Sarno, A., Mettivier, G., Golosio, B., Oliva, P., Spandre, G., Di Lillo, F., Fedon, C., Longo, R. \& Russo, P. (2016a). Phys. Med. 32, 681690.

Sarno, A., Mettivier, G. \& Russo, P. (2015). Med. Phys. 42, 2786-2804. Stevenson, A. W., Crosbie, J. C., Hall, C. J., Häusermann, D., Livingstone, J. \& Lye, J. E. (2017). J. Synchrotron Rad. 24, 110-141.

Stevenson, A. W., Hall, C. J., Mayo, S. C., Häusermann, D., Maksimenko, A., Gureyev, T. E., Nesterets, Y. I., Wilkins, S. W. \& Lewis, R. A. (2012). J. Synchrotron Rad. 19, 728-750.

Stevenson, A. W., Mayo, S. C., Häusermann, D., Maksimenko, A., Garrett, R. F., Hall, C. J., Wilkins, S. W., Lewis, R. A. \& Myers, D. E. (2010). J. Synchrotron Rad. 17, 75-80.

Taylor, C., Correa, C., Duane, F. K., Aznar, M. C., Anderson, S. J., Bergh, J., Dodwell, D., Ewertz, M., Gray, R., Jagsi, R., Pierce, L., Pritchard, K. I., Swain, S., Wang, Z., Wang, Y., Whelan, T., Peto, R., McGale, P. \& Early Breast Cancer Trialists' Collaborative Group (2017). J. Clin. Oncol. 35, 1641-1649.

Torre, L. A., Siegel, R. L., Ward, E. M. \& Jemal, A. (2015). Cancer Epidemiol. Biomarkers Prev. 25, 16-27.

Verma, V., Shah, C. \& Mehta, M. P. (2016). Clin. Breast Cancer, 16, 145-154.

Wysokinski, T., Harkness, T., Belev, G., Dawicki, W., Arnason, T., Gordon, J., Davied, G., Lobanova, L., Zhu, N., Webb, A., Miller, D., Chapman, D. \& Liu, X. (2016). Proceedings of the 39th Canadian Medical and Biological Engineering Conference (CMBEC39), 24-27 May 2016, Calgary, Alberta, Canada. 